\title{
Clinical heterogeneity and unusual presentations of Creutzfeldt-Jakob disease in Jewish patients with the PRNP codon 200 mutation
}

\author{
J Chapman, P Brown, L G Goldfarb, A Arlazoroff, D C Gajdusek, A D Korczyn
}

\begin{abstract}
The cluster of Creutzfeldt-Jakob disease among Jews of Libyan origin is the largest in the world. It was found that the disease in this ethnic group is linked to a point mutation in codon 200 of the prion protein precursor gene. In this study the clinical data from 14 such patients are described, demonstrating wide phenotypic heterogeneity. The age of onset ranged from 34 to 65 years and the duration of disease from 2 to 66 months. Clinical features included cerebral, basal ganglia, brainstem, cerebellar, and spinal cord dysfunction. Uncommon features included fatal insomnia in one patient, pruritus in another, and demyelinating peripheral neuropathy in two.
\end{abstract}

$(\mathcal{F}$ Neurol Neurosurg Psychiatry 1993;56:1109-1112)

Creutzfeldt-Jakob disease (CJD) is a rare cause of progressive dementia. Recent advances in the understanding of the molecular biology of spongiform encephalopathies have revealed that many, or perhaps all, familial cases of CJD and GerstmannStraussler-Scheinker disease (GSSD) are linked to specific point mutations in the prion protein precursor gene (PRNP). ${ }^{1}$ Different mutations of the PRNP gene seemingly have distinguishing clinical features. For example, a mutation at codon 102 leads to GSSD, ${ }^{2}$ another at codon 200 is linked to CJD, ${ }^{3}$ while a mutation at codon 129 is clinically silent or confers susceptibility to prion infection. ${ }^{4}$ Although CJD is usually sporadic, a cluster of patients was described in Israel among Jews of Libyan origin..$^{5-9}$ It was first suggested that ingestion of scrapie-infected sheep brains accounted for this cluster, ${ }^{10}$ although evidence of familiality existed. ${ }^{11}$ Further data cast serious doubt on the infectious transmission of the disease in this ethnic group,,$^{12}$ and indeed, we have demonstrated that CJD in Jewish patients of Libyan origin is associated with a $G$ to A (glutamic acid to lysine) mutation at codon 200 of the PRNP gene. ${ }^{13}$ This finding was later confirmed by others. ${ }^{14}$ The accumulated data have led to much speculation on the pathogenesis of the disease in genetic as opposed to sporadic CJD cases. ${ }^{15}$ Previous studies of Libyan Jewish patients included cases diagnosed on the basis of classic neurological features. The availability of molecular biology tools now allows for recog- nition of cases based on genetic screening. It is therefore of interest to delineate the spectrum of clinical manifestations of $\mathrm{CJD}$ in patients with a specific point mutation. We now describe clinical data from 14 CJD Jewish patients of Libyan, Tunisian, and Greek origin who carried the PRNP codon 200 mutation, demonstrating a wide spectrum of clinical heterogeneity.

\section{Materials and methods}

All patients were in the department of neurology, Tel-Aviv Medical Center, except for patients 6,10 , and 13 who were examined by one of us (JC or AA) and whose hospital records were reviewed. A full history was obtained for each patient including genealogical data. Patients were followed up through most of the clinical course of the disease. All were screened by $C B C$, blood chemistry, $B_{12}$, $\mathrm{FT}_{4}, \mathrm{VDRL}$, urine and CSF studies, blood and urine cultures, chest $x$ rays, CT of the brain, and repeated EEG examinations. Electromyographic studies were performed in six cases. In nine cases the diagnosis of CJD was confirmed by either brain biopsy or necropsy. The codon 200 mutation was identified in all cases as previously described. ${ }^{913}$

\section{Results}

The table presents clinical data from the 14 patients, nine males and five females. Most patients were Jews born in Libya, but case 1 was born in Greece of Jewish parents who did not have any apparent links to Libyan Jews. Patients 8 and 9 were born in Tunisia but had Libyan ancestors while patients 11-13 were born in Libya of one Libyan ancestor, the other being either Tunisian or Turkish. All cases were considered familial in that at least one family member had previously died of a disease compatible with CJD. All patients were heterozygous for the PRNP codon $200^{\text {Lys }}$ mutation except for case 14 who is homozygous for this mutation. The median age of onset of the disease was 53 years with a range of 34 to 65 years. Some of the patients had a prodromal syndrome consisting of generalised weakness and malaise. Fever above $39^{\circ} \mathrm{C}$, not accompanied by leucocytosis or an obvious infectious cause and unresponsive to antibiotics, occurred in 10 cases in the later stages of the disease.

Cognitive deterioration included memory disturbances in 13 patients as well as visual hallucinations and delusions. Other mental 
Table Summary of clinical data on 14 mutation positive CFD patients

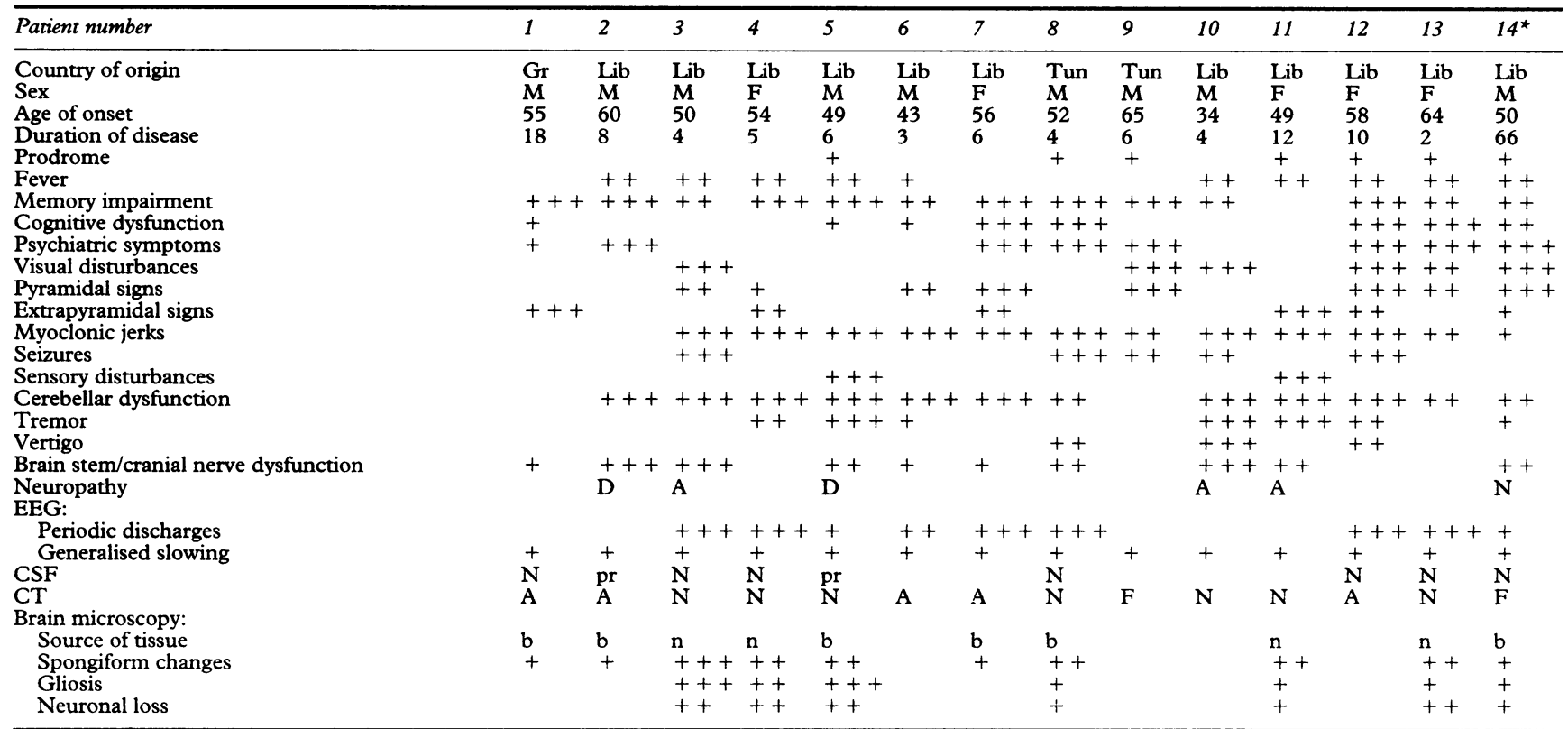

The country of origin of the patients is indicated as Libya ( $\mathrm{Lib}$ ), Tunisia (Tun), or Greece (GR) and sex indicated as male (M) or female (F). Age of onset is given in years and duration of disease in months. A prodrome consisted of generalised weakness and malaise. Fever is indicated when no infectious cause was found. Cognitive dysfunction indicates signs such as apraxia, agnosia, etc and psychiatric symptoms included psychosis or depression. Pyramidal signs consisted of weakness and spasticity, and extrapyramidal signs included rigidity and bradykinesia. Cerebellar dysfunction included limb and gait ataxia. Brain stem and cranial nerve ness and spasticity, and extrapyramidal signs included rigidity and bradykinesia. Cerebellar dysfunction included limb and gait ataxia. Brain stem and cranial nerve
impairment produced dyphagia, dysphonia, dysarthria, and facial palsy. Neuropathy was either demyelinating (D) or axonal (A) neuropathy as indicated by nerve impairment produced dyphagia, dysphonia, dysarthria, and facial palsy. Neuropathy was either demyelinating (D) or axonal (A) neuropathy as indicated by nerve
conduction and electomyographic studies (which were normal in the patient marked $N$ ). Electroencephalographic findings (EEG) refer to periodic discharges and conduction and electomyographic studies (which were normal in the patient marked N). Electroencephalographic findings (EEG) refer to periodic discharges and
generalised slowing. Findings in the CSF are presented as $\mathrm{N}$, indicating normal biochemistry and microscopy, or pr, indicating elevated protein levels (70 and 160 generalised slowing. Findings in the CSF are presented as $\mathrm{N}$, indicating normal biochemistry and microscopy, or pr, indicating elevated protein levels (70 and 160
$\mathrm{mg} / 100 \mathrm{ml}$ respectively). CT findings are represented as either normal (N), generalised atrophy (A), or focal hypodense lesions ( $\mathrm{F}$ ). Brain microscopy of tissue from either a biopsy (b) or necrospy ( $n$ ) are detailed as findings of spongiform changes, gliosis, and neuronal loss.

+++ indicates symptoms occurring in the initial stages, ++ and + indicate severe and mild symptoms occurring late in the course of the disease respectively.

*indicates the patient, who is homozygous for the PRNP mutation, is still alive, 66 months after onset.

disturbances included aggressive behaviour, psychosis, and depression. Signs of localised cortical dysfunctions such as apraxia and agnosia were also seen. Patient 13 presented with insomnia which was complete for at least one week and was accompanied by bulimia and agitation. She then lapsed into coma over a period of a few hours and died 1 month later. Patient 11, a 49 year old woman, was alert and did not seem to have overt cognitive deficits until severe bulbar involvement necessitated intubation and positive pressure ventilation which made evaluation of her higher mental function difficult. This patient was alert until she died suddenly of pulmonary oedema 12 months after the onset, four months following intubation; this was taken to be of central origin. ${ }^{16}$ All other patients were in coma preceding their death.

Brainstem and cranial nerve impairment producing dysphagia, dysphonia, dysarthria, and facial palsy was found in nine patients and dizziness and vertigo in three. Cerebellar dysfunction, including gait and limb ataxia, occurred in 12 patients. Visual disturbances in five patients included visual hallucinations, cortical blindness, and optic ataxia $(1,2$, and 3 cases respectively). Pyramidal signsspastic weakness, exaggerated tendon reflexes, and extensor plantar responseswere found in seven patients and were symmetrical except for patient 9, who suffered a stroke during the course of the disease, and patient 14. Extrapyramidal signs manifesting as cogwheel rigidity and bradykinesia were found in five cases. Eleven patients had myoclonic jerks, and five had generalised tonic-clonic seizures. Patients 3 and 6 had a startle response to noise and touch in addition to spontaneous myoclonic jerks. Sensory disturbances were found in patient 5 who had peripheral neuropathy. Patient 11 presented with severe pruritus which persisted throughout the course of the disease and was accompanied by a dermal rash with an inflammatory cellular infiltration of the skin. Evidence of peripheral neuropathy was found in five cases by clinical examination and confirmed electrophysiologically. Patients 2 and 5 had evidence of a demyelinating neuropathy which was described in detail elsewhere ${ }^{17}$ while the other three patients had motor axonal neuropathy. The two patients with demyelinating polyneuropathy had elevated levels of protein in the CSF, but in the others normal protein, cell, and glucose CSF content were found.

Electroencephalographic findings included slowing of the background activity in all the patients and periodic discharges in eight. The generalised pattern of discharges seen in routine EEGs was clearly shown on brain mapping to be not strictly generalised on onset nor bilaterally symmetrical. ${ }^{18} \mathrm{CT}$ studies of the brain were normal in six and showed generalised atrophy in six. In cases 9 and 14 focal cerebral lesions compatible with infarcts were seen on CT. Biopsy or postmortem examination of the brain was performed in nine of the patients and, although the diagnosis of CJD was confirmed in all these cases, the severity of the pathological changes varied widely, 
possibly reflecting the brain areas affected and those sampled, as well as the stage of the disease at the time of biopsy.

\section{Discussion}

We have summarised the clinical findings in 14 Jewish CJD patients of Libyan, Tunisian, and Greek origin carrying the codon 200 mutation in the PRNP gene. The PRNP codon 200 point mutation is prevalent in Jews of both Libyan and Tunisian origin reflecting the close ties that existed between these two communities. How the mutation reached the Jewish community in Greece (Saloniki) is unclear, although some trading links with north Africa probably existed. It is interesting to note that the codon 200 mutation has also been identified in Slovakia and in Chile. ${ }^{19}$ It is still unclear to what extent these represent independent mutations or genetic drift. Speculations were raised that a single mutation occurred in Spain before the Jews were expelled in 1492 . However, cases of this mutation are yet to be described in Spain itself. In addition, the recent finding of an identical mutation in Sicily (Korczyn and Chapman, unpublished results) and particularly in Japan ${ }^{20}$ do not seem to fit in with this theory.

Initial clinical reports of CJD patients in Israel included five out of 12 of Jewish Libyan origin $^{56}$ and, although our general understanding of the pathogenesis of this cluster has greatly advanced since then, there have been few detailed descriptions of such patients. Earlier reports linking the PRNP codon 200 mutation to the disease did not include detailed clinical descriptions. ${ }^{114}$ Recently, a comparison of clinical findings in 49 Libyan patients with 65 non-Libyan CJD patients in Israel was published. ${ }^{21}$ However, it was not specified in this study which patients were actually positive for the codon 200 mutation. In addition, Israeli-born patients of Libyan origin and patients of Tunisian, Egyptian, and Greek origin who may well have had the mutation were included in the non-Libyan group. In contrast, all patients in the current report definitely carried this mutation. The existence of this marker for the disease enabled the recognition of atypical forms which may not have been clinically diagnosed as CJD, such as patients $9,10,11$, 13, and 14. This factor may account for the heterogeneity of the clinical findings including age of onset, duration of the disease, and the presence or absence of cerebral, basal ganglionic, brainstem, cerebellar, and spinal cord dysfunction. The clinical heterogeneity of the codon 200 point mutation is especially evident in patients presenting with clinical features not usually associated with $\mathrm{CJD},{ }^{22}$ such as peripheral neuropathy, pruritus, insomnia, or protracted dementia without other distinguishing characteristics.

Considerable phenotypic heterogeneity has been demonstrated in many of the forms of inherited prion disease, in particular those associated with base pair insertions (144) where the phenotypic range within a single kindred is extraordinarily large..$^{23}{ }^{24}$ It is interesting to note that when cases are obtained from genetic screening, rather than simply looking for mutations in cases preselected on the basis of their classic clinical appearance, similar clinical heterogeneity is demonstrated in the codon 200 mutation. This finding underlines yet again the need to establish the full range of these disorders by genetic screening of neurodegenerative diseases.

The case of fatal insomnia is of interest because of the recently described association of the PRNP $178^{\text {Asn }}$ mutation with fatal familial insomnia. ${ }^{25} 26$ We are currently performing histological studies on this patient's brain in order to determine the existence of thalamic pathology associated with fatal familial insomnia. However, it should be recalled that insomnia has been described in a classic case of $\mathrm{CJD} .{ }^{27}$ Another striking feature is the prolonged course of disease in the single patient homozygous for the $200^{\text {Lys }}$ allele. A patient previously described as homozygous for this mutation had a much shorter duration cf disease that was clinically indistinguishable from that affecting heterozygous carriers, and this was taken to imply that this point mutation is a "true dominant" disease. ${ }^{14}$ However, the unusually long course in our homozygous patient raises other possibilities. More homozygous patients must be examined in order to better define whether they have distinct clinical features. Such carriers could exist since the Libyan cluster of CJD is relatively large and there is a significant proportion of intermarriage among potential carriers.

Several other dominantly inherited diseases demonstrate a significant diversity in clinical features and age of onset. In some cases, like myotonic dystrophy, the underlying molecular mechanisms were recently clarified ${ }^{28}$ and may be similar to the molecular heterogeneity associated with insertions in the PRNP gene. ${ }^{29} \mathrm{~A}$ wide clinical range may, however, also occur in diseases with a presumably single and identical point mutation such as torsion dystonia in Ashkenazi Jews, ${ }^{30}$ and is also obvious in this relatively small group of patients with CJD. Recently it has been shown that additional polymorphisms on the PRNP gene may influence the phenotypic expression of the $178^{\text {Asn }}$ mutation. ${ }^{31} \mathrm{We}$ are currently examining the PRNP gene of our patients in order to determine the existence and relevance of other polymorphisms. Nongenetic factors may also influence the age of onset or the clinical progression of the disease; elsewhere we have suggested the involvement of female sex hormones (Chapman and Korczyn, submitted for publication) but other factors are not obvious. We have also identified nine cases of elderly carriers of the PRNP $200^{\text {Lys }}$ mutation who had dementia for several years without distinguishing features. It is unknown whether these were asymptomatic carriers with incidental Alzheimer's disease or prion dementia. ${ }^{23}$ Prospective studies on large num- 
bers of presymptomatic carriers of the codon 200 mutation are necessary in order to address this question of genetic or other modifying factors. Identification of such factors will deepen our understanding of the pathogenesis of the disease and may eventually provide a therapeutic strategy.

1 Hsiao K, Prusiner SB. Inherited human prion diseases. Neurology 1990;40:1820-7.

2 Hsiao K, Baker HF, Crow TJ, et al. Linkage of a prion protein missense variant to Gerstmann-Straussler synprotein missense variant to Gerst

3 Goldgaber D, Goldfarb LG, Brown P, et al. Mutations in familial Creutzfeldt-Jakob disease and Gerstmannfamilial Creutzfeldt-Jakob disease and Gerstmann204-6.

4 Collinge J, Palmer MS, Aidan JD. Genetic predisposition to iatrogenic Creutzfeldt-Jakob disease. Lancet 1991 337:1441-2.

5 Behar M, Sroka C, Elian M, et al. Creutzfeldt-Jakob disease and its relation to senile dementia. Harefuah 1969 77:275-9.

6 Goldhammer Y, Bubis JJ, Sarova-Pinhas I, Braham J. Subacute spongiform encephalopathy and its relation to Jakob-Creutzfeldt disease: report on six cases. $\mathcal{F}$ Neurol Neurosurg Psychiatry 1972;35:1-10.

7 Kahana E, Alter M, Braham J, Sofer D. Creutzfeldt-Jakob disease: focus among Libyan Jews in Israel. Science 1974;183:90-1.

8 Korcyzn AD. Creutzfeldt Jakob disease among Libyan Jews. Eur f Epidemiol 1991;7:490-3.

9 Chapman J, Korczyn AD. Genetic and environmental factors determining the development of Creutzfeldt-Jakob disease in Libyan Jews. Neuroepidemiology 1991;10. 228-31.

10 Alter M. Creutzfeldt-Jakob disease: hypothesis for high incidence in Libyan Jews in Israel. Science 1974; 186:848.

11 Neugut RH, Neugut AI, Kahana E, Stein Z, Alter M Creutzfeldt-Jakob disease: familial clustering among Libyan-born Israelis. Neurology 1979;29:225-31.

12 Nisipeanu $P, E l$ Ad B, Korczyn AD. Spongiform encephalopathy in an Israeli born to immigrants from Libya. Lancet 1990;336:686.

13 Goldfarb LG, Korczyn AD, Brown P, Chapman J, Gajdusek DC. Mutation in codon 200 of scrapic amyloid precursor gene linked to Creutzfeldt-Jakob disease in Sephardic Jews of Libyan and non-Libyan origin Lancet 1990;336:637-8.

14 Hsiao K, Meiner Z, Kahana E, et al. Mutation of the prion protein in Libyan Jews with Creutzfeldt-Jakob disease. $N$ Engl f Med 1991;324:1091-7.

15 Will RG. The spongiform encephalopathies. I Neurol Neurosurg Psychiatry 1991;54:761-3.

16 Rock M, Korczyn AD. Neurogenic pulmonary edema. Pract Cardiol 1984;10:99-103.

17 Neufeld MY, Josiphov J, Korczyn AD. Peripheral demyelinating neuropathy in Creutzfeldt-Jakob disease. demyelinating neuropathy in

18 Neufeld MY, Korczyn AD. Topographic distribution of the periodic discharges in Creutzfeldt-Jakob disease (CJD). Brain Topography 1992;4:201-6.

19 Goldfarb LG, Brown P, Mitrova E, et al. CreutzfeldtJacob disease associated with the PRNP codon $200^{L_{3}}$ mutation: an analysis of 45 families. Eur $\mathcal{f}$ Epidemio 1991;7:477-86.

20 Kitamoto T, Doh-ura K, Tateishi J. Primary structure of prion protein influences clinical and pathological aspects of Creutzfeldt-Jakob disease Neurobiol Aging 1992;13(suppl 1): S90.

21 Kahana E, Zilber N, Abraham M. Do Creutzfeldt-Jakob disease patients of Jewish Libyan origin have unique disease patients of Jewish Libyan origin

22 Brown $P$. The clinical neurology and epidemiology of Creutzfeldt-Jakob disease: with special reference to Creutzfeldt-Jakob disease: with special reference to iatrogenic cases. In: Bock $G$, Marsh J, eds. Novel infectious agents and the central nervous system. (CIBA Foun 23 .

23 Collinge J, Harding AE, Owen $\mathrm{F}$, et al. Diagnosis of Gerstmann-Straussler syndrome in familial dementia with prion protein gene analysis. Lancet 1989;2:15-7.

24 Collinge J, Owen F, Poulter $\mathrm{H}$, et al. Prion dementia without characteristic pathology. Lancet 1990;336:7-9.

25 Lugaresi E, Medori R, Montagna P, et al. Fatal familial insomnia and dyautonomia with selective degeneration of thalamic nuclei. $N$ Engl $F$ Med 1986;315:997-1003.

26 Medori R, Tritchler H-J, LeBlanc A, et al. Fatal familia insomnia, a prion disease with a mutation at codon 178 of the prion protein gene. $N$ Engl $f$ Med 1992;326: of the

27 Nevin S, McMenemey WH, Behrman S, Jones DP. Subacute spongiform encephalopathy - a subacute form of encephalopathy attributable to vascular dysfunction (spongiform cerebral atrophy). Brain 1960;83:519-63.

28 Rosenberg RN. Amplification signals anticipation-les DNA is better. Neurology 1992;42:1857-8.

29 Goldfarb LG, Brown P, McCombie WR, et al. Transmissible familial Creutzfeldt-Jakob disease associated with five, seven, and eight extra octapeptide coding repeats in the PRNP gene. Proc Natl Acad Sci USA 1991;88:10926-30.

30 Korczyn $A D$. Genetics of idiopathic torsion dystonia in Ashkenazi Jews. In: Bonne-Tamir B, Adam A, eds. Genetic diversity among fews. New York and Oxford: Genetic diversity among fews. New York

31 Goldfarb LG, Peterson RB, Massimo T, et al. Fatal familial insomnia and familial Creutzfeldt-Jakob disease: disial insomnia and familial Creutzfeldt-Jakob disease: disScience 1992;258:806-8. 